\title{
Computerassistierte \\ Präzisionschirurgie am Ohr
}

\author{
Computer-Assisted Precision Surgery in the Ear
}

Brett Bell, Nicolas Gerber, Kate Gavaghan, Stefan Weber, Institut für chirurgische Technologien und

Biomechanik, Universität Bern,

Christoph Stieger, ARTORG Zentrum für Biomedizinische Forschung, Universität Bern,

Marco Caversaccio, Universitätsklinik für Hals-, Nasen- und Ohrenkrankheiten, Inselspital Bern

Zusammenfassung Chirurgische Eingriffe am Ohr stellen aufgrund der komplexen Anatomie und der Grössenverhältnisse der beteiligten anatomischen Strukturen eine Herausforderung für den HNO-Chirurgen dar. In diesem Beitrag wird ein Ansatz für die roboterbasierte Navigation zur Hörgeräteimplantation vorgestellt. Insbesondere wird auf die Möglichkeit des Fräsens von Implantatlagern im Felsenbein eingegangen. Je präziser ein Implantat im Schädel verankert werden kann, desto einfacher ist der chirurgischen Ablauf. Weiterhin, profitieren Patienten von verkürzten Operationszeiten und weniger schmerzhaften Eingriffen. $\gg$ Summary Traditional surgical procedures involving the implantation of artificial hearing devices in the inner ear are challenging due to the size and complexity of anatomical structures within the temporal bone. To date, no stereotactic instrument guidance technology providing the necessary levels of accuracy is available. This work presents an approach to robot assisted implantation of hearing devices. Specifically, the robot system was used to mill a cavity to for a direct acoustical stimulation implant. As the precision of such cavities increases, so also can future implant generations improve in terms of size, complexity and cost effectiveness. Additionally, patients themselves would profit from shorter procedure times and less painful interventions.

Schlagwörter Hörgeräteimplantation, chirurgische Navigation, Robotik $\gg$ Keywords Hearing aid implantation, surgical navigation, robotics

\section{Einführung}

\subsection{Implantation von Hörgeräten}

Hörgeräte werden seit mehr als drei Jahrzehnten kontinuierlich weiterentwickelt und erfolgreich in der $\mathrm{Pa}-$ tientenversorgung eingesetzt. Derzeit können aber noch immer nicht alle in Frage kommenden Patienten mit einem entsprechenden Hörverlust behandelt werden, selbst wenn derzeit verfügbare Implantattechnologien eigentlich ein verbessertes Sprachverständnis ermöglichen würden. Ein möglicher Grund dafür ist der komplexe chirurgische Ablauf der Implantation, der die Entfernung eines Teils des Felsenbeins erfordert (sogenannte Mastoidektomie). Im Felsenbein verlaufen unter anderem der Gesichtsnerv
(N. Facialis) und die Paukensaite ${ }^{1}$ (Chorda Tympani). Um Verletzungen an diesen Strukturen zu vermeiden, müssen diese während des Eingriffes sicher identifiziert und geschützt werden. Dazu wird das Mastoid grösser ausgefräst, als für die eigentliche Platzierung des Implantats erforderlich wäre.

Der bevorzugte Zugang zum Mittelohr und zur Cochlea führt durch eine schmale Öffnung zwischen Gesichtsund Geschmacksnerv hindurch (facial recess, siehe Bild 1). Der Gesichtsnerv bildet dabei die posterior-superiore

\footnotetext{
${ }^{1}$ Die Paukensaite ist ein Ast des Nervus facialis und unter anderem für den Geschmackssinn zuständig.
} 


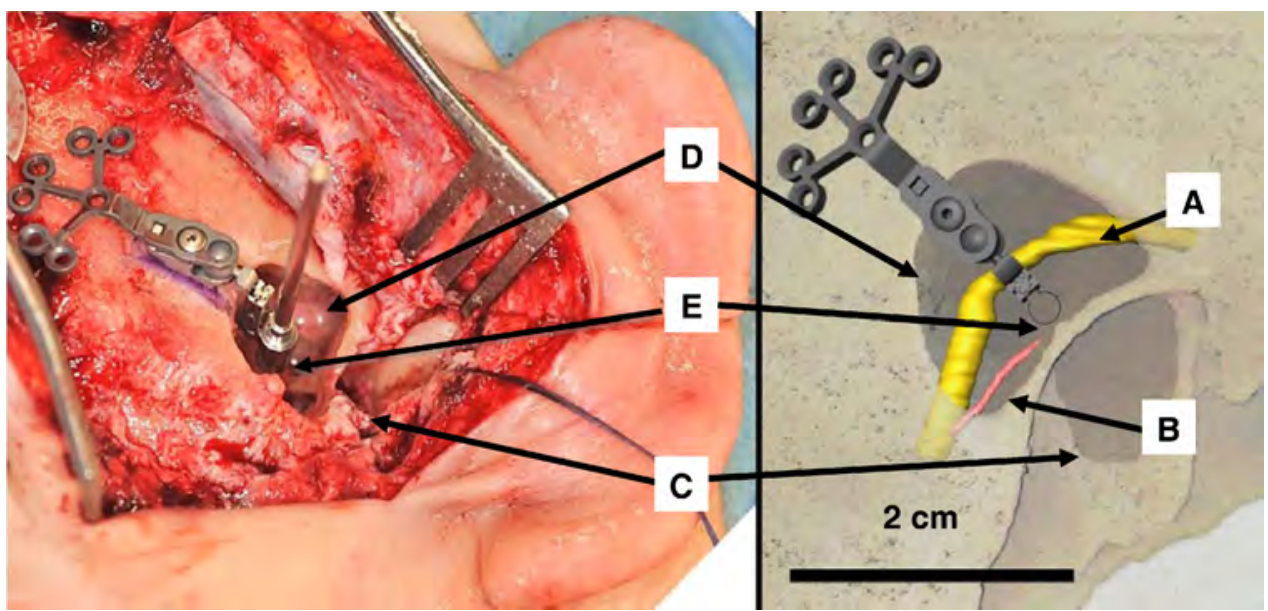

Bild 1 Darstellung der Mastoidektomie und einem implantiertem elektromechanischen Hörgerät. Dargestellt sind die ungefähre Lage des Gesichtsnervs (A), der Paukensaite (B), des äusseren Gehörgangs (C), der Masoidektomie (D), und der Durchgang zum Innenohr (E).

Grenze, Paukensaite und äusserer Gehörgang die inferioranteriore Grenze. Der für den Durchstoss zum Innenohr verfügbare Platz beträgt dabei nur wenige Millimeter. Um eine Verletzung dieser sensiblen Strukturen während eines instrumentengeführten (also navigierten) Fräs- und Bohrvorgangs (typischerweise mit einem Bohrer von ca. 1,5 mm Durchmesser) zu vermeiden, ist daher eine Präzision des technischen Gesamtprozesses von unter 0,5 $\mathrm{mm}$ erforderlich [1]. Dieses Präzisionsniveau liegt fast um den Faktor zehn unter der Genauigkeit kommerziell verfügbarer stereotaktischer Instrumentenführungssysteme. Auf klinischer Seite stehen damit keine Erfahrungen beim Einsatz von Führungssystemen zur Verfügung.

\subsection{Instrumentenführungsysteme für die Chirurgie am Ohr}

Stereotaktische Instrumentenführung (im Folgenden als Navigationssysteme bezeichnet) wird eingesetzt, um durch eine verbesserte Orientierung und Lokalisation im Situs die chirurgische Umsetzung eines geplanten Eingriffs zu optimieren. Damit können unter Umstän- den weitere Effekte wie Reduzierung der Operationszeit, Reduzierung der Invasivität und Verbesserung des Operationsergebnisses erreicht werden. Diese Systeme erfassen dabei die Position und Orientierung von chirurgischen Instrumenten und anatomischen Strukturen (also Teilen des Patienten) im Raum ${ }^{7}$. Die Lage der erfassten Instrumente relativ zum Patienten wird an einem Bildschirm und im Kontext der prä- oder intraoperativ erhobenen 2D/3D/4D Bilddaten angezeigt. Variationen dieser Systeme beziehen sich dabei auf das chirurgische bzw. interventionelle Einsatzgebiet, die Art und Weise der Referenzierung zwischen Bilddaten und Patient (Registrierung) und die verwendeten Instrumente. In [2] findet sich ein Überblick über den aktuellen Stand der Technik bei stereotaktischen Navigationssystemen. Typische Fehlereinflüsse auf die erreichbare Gesamtgenauigkeit bei der Verwendung derartiger Instrumentenführungssysteme stellen dabei die Bildgebung (typischerweise

${ }^{7}$ Dies geschieht typischerweise über optische oder elektromagnetische Messverfahren.

Tabelle 1 Vergleich der Einflüsse wichtiger Fehlerquellen zwischen verfügbaren Navigationssystemen in der computerassistierten Chirurgie (CAS) und das notwendige Genauigkeitsniveau in der Mikrochirurgie (CAMS).

\begin{tabular}{|c|c|c|c|c|c|}
\hline $\begin{array}{l}\text { Fehlerquelle } \\
\text { Typ } \\
\text { Einheit }\end{array}$ & $\begin{array}{l}\text { Bildgebung } \\
\text { Voxel }^{2} \\
{[\mathrm{~mm}]}\end{array}$ & $\begin{array}{l}\text { Positionsmessung } \\
\text { RMS }^{3} \\
{[\mathrm{~mm}]}\end{array}$ & $\begin{array}{c}\text { Registrierung } \\
\text { TFE }^{4} \\
{[\mathrm{~mm}]}\end{array}$ & $\begin{array}{l}\text { Positionierung } \\
\text { RMS }^{5} \\
{[\mathrm{~mm}]}\end{array}$ & $\begin{array}{c}\text { Gesamtfehler } \\
\text { TRE }^{6} \\
{[\mathrm{~mm}]}\end{array}$ \\
\hline CAS & 0,60 (CT) & 0,25 & $1-2$ & $0,5-2,0$ & $2-3$ \\
\hline CAMS & 0,15 (DVT) & 0,10 & $0,1-0,2$ & 0,1 & 0,5 \\
\hline
\end{tabular}

\footnotetext{
${ }^{2}$ Grösste Voxeldimension in 3D Bilddaten (falls nicht isometrisch);

${ }^{3}$ RMS der Positionsmessung: entspricht der mittleren Abweichung der vom optischen Messsystem angegebenen Position vom jeweiligen Mittelwert

${ }^{4}$ FRE: Fiducial Registration Error ist der Fehler als Ergebniss des jeweils angewandten Registrierverfahrens zwischen Bilddaten und Patient auf der Basis von definierten Landmarken;

${ }^{5}$ RMS der Positionierung: mittlerer Fehler des Roboter bzw. der Hand des Chrirurgen beim Einstellen einer definierten Sollposition;

6 TRE: Target Registration Error ist die Abweichung zwischen erreichter Position/Orientierung und Sollposition bzw. -orientierung. Der TRE ist die Vereinigung aller auftretenden Fehlerquellen im Behandlungsprozess. (der TRE kann nur über zusätzliche postoperative Bildgebung ermittelt werden, was klinisch teilweise wegen der zusätzlichen Strahlenbelastung nicht möglich ist).
} 
bedingt durch die begrenzte Auflösung des Verfahrens und Verzerrungen in den Bilddaten), die Genauigkeit des verwendeten Positionsmessverfahrens (geometrische Genauigkeit, Messrauschen und Stabilität gegenüber äusseren Einflüssen) und Registrierung (bedingt durch Art des Verfahrens, Anzahl und Art der verwendeten Landmarken). Diese Fehler resultieren in einem geometrischen Gesamtfehler eines Navigationsverfahrens und stellen die Summe aller im Workflow vorhandenen Teilfehlerquellen dar. In Tabelle 1 sind die zu erwartenden Grössenordnungen für die Teilfehlerquellen im Vergleich zwischen derzeit verfügbaren CAS-Systemen und dem notwendigen Genauigkeitsniveau in der Mikrochirurgie dargestellt. Im Vergleich $\mathrm{zu}$ den klinisch verfügbaren Navigationsansätzen ist es Ziel dieser Arbeiten, den Gesamtfehler bei der Instrumentenpositionierung auf einen Wert unter $0,5 \mathrm{~mm}$ zu senken und damit das Prinzip der computerassistierten Mikrochirurgie (CAMS) [6] am Ohr zu ermöglichen.

\subsection{Chirurgische Robotersysteme für die Chirurgie am Ohr}

Derzeit verfügbare Verfahren und Ansätze der stereotaktischen Navigation sind für den Einsatz in der Mikrochirurgie ungeeignet, weil sie die erforderliche Gesamtgenauigkeit von $<0,5 \mathrm{~mm}$ nicht erreichen können. Ein weiterer Nachteil bei der Verwendung zwar navigierter aber dennoch freihandgeführter Instrumente in der Mikrochirurgie besteht zudem in der fehlenden Präzision beim Erreichen sehr kleiner anatomischer Strukturen und die fehlende Kompensation von unerwünschten Zitterbewegungen des Operateurs (Tremor). Für mikrochirurgische Verfahren können daher zusätzliche mechanische Führungssysteme eingesetzt werden, die ohne unerwünschte Handbewegungen und mit der erforderlichen Genauigkeit mikrochirurgische Manipulationsaufgaben präzise ausführen können wie beispielsweise das Fräsen eines minimalinvasiven Zugangs im Mastoid.

Eine weitere Möglichkeit der Instrumentenführung ist die Verwendung chirurgischer Roboter. Diese haben den Vorteil dass sowohl beliebige Trajektorien gebohrt als auch komplexe Kavitäten gefrässt werden können. Erste Anwendungen robotischer Instrumentenführungssystems für die Eingriffe am Ohr wurden auf Basis von Industrierobotern beschrieben [6-8]. Hierbei wird der Roboter in Kombination mit optischen Navigationssystemen eingesetzt um präzise Fräsvorgänge am Mastoid zu ermöglichen. Diese Verfahren verwenden Knochenschrauben als künstliche Landmarken, die bereits $\mathrm{zu}$ einem Zeitpunkt vor der 3D-Bildgebung implantiert werden. Die Landmarken werden in den Bilddaten identifiziert und vor dem eigentlichen Bohren als Referenzpunkte durch das Robotersystem [6] oder durch das Navigationssystem $[4 ; 5]$ eingemessen und die Positionen am Patienten dann mit den Schraubenpositionen in den Bilddaten referenziert. Der Roboter führt dann die vorher definierten Bohr- und Fräsaufgaben automatisch oder interaktiv aus bzw. unterstützt den Chirurg. Die maximal erreichbare Präzision dieser Verfahren ist typischerweise durch die Genauigkeit das verwendeten Registrierverfahrens und die Genauigkeit des verwendeten geometrischen Messverfahrens (optisches Messverfahren, mechanisch über Roboter) limitiert. Die genannten Arbeiten erreichen teilweise bereits die klinisch relevante Präzision von $0,5 \mathrm{~mm}$, aber die vorhandene Standardabweichung ist aus sicherheitstechnischer Sicht noch zu hoch. Sowohl der Platzverbrauch als auch der notwendige Aufwand beim Aufbau der Robotersysteme im OP sowie die erforderlichen Adaptionen des etablierten klinischen Workflows limitieren bisher den Einsatz von Industrierobotern im OP.

\section{Roboterbasierte Instrumentenführung für die Chirurgie am Ohr}

Im vorliegenden Beitrag wird der Einsatz des Robotorsystems für das Fräsen einer Implantattasche vorgestellt. Diese Tasche nimmt den Stimulator eines elektromechanischen Stimulationsimplantats (Direct Acoustical Cochlear Stimulation, DACS $)^{8}$ auf. Dazu wurde ein bereits existierendes roboterbasiertes Navigationssystem verwendet, dessen Aufbau im folgenden Kapitel beschrieben wird. Die klinische Praktikabilität des Verfahrens als grundlegende Anforderung für eine spätere Überführbarkeit dieses Ansatzes in die klinische Praxis wird ebenfalls diskutiert.

\subsection{Robotersystem}

Es wurde ein serieller Knickarmroboter mit fünf Freiheitsgraden entwickelt. Aus Komplexitäts- und Gewichtsgründen wurde auf zusätzliche Freiheitsgrade, die Rotationen um die Instrumentenachse (6. Freiheitsgrad) bzw. das Erreichen einer Position in verschiedenen Konfigurationen (7. Freiheitsgrad) verzichtet. Die geometrische Größe des Roboters und die daraus ableitbaren Parameter für die Armlänge der einzelnen Gelenke sowie für den verfügbaren Arbeitsraum wurden zu Projektbeginn auf der Basis von Rapid-Prototyping Modellen im OP ermittelt und optimiert. Insbesondere musste die Anwesenheit eines Operationsmikroskops im Situs berücksichtigt werden. Daher wurde eine serielle Kinematik gewählt, die eine Positionierung der Roboterbasis seitlich vom Kopf und von dort ein seitliches Einschwenken des Arms in den Situs ermöglicht. Der optimale Arbeitsraum befindet sich demnach in dem Bereich, in dem Achsen 2 und 3 jeweils um $90^{\circ}$ zueinander eingeschwenkt sind (vergleiche Bild 2 links). Darüber hinaus wurde das maximale Gewicht des Robotersystems auf $5 \mathrm{~kg}$ festgelegt, um einen manuellen Aufbau durch das OP-Personal zu ermöglichen.

Das geringe Gewicht erlaubt gleichzeitig die Montage des Roboters direkt am OP-Tisch, wodurch relative Bewegungen zwischen Patient und Roboterbasis minimiert

${ }^{8}$ DACS - Direct Acoustic Cochlea Stimulation 


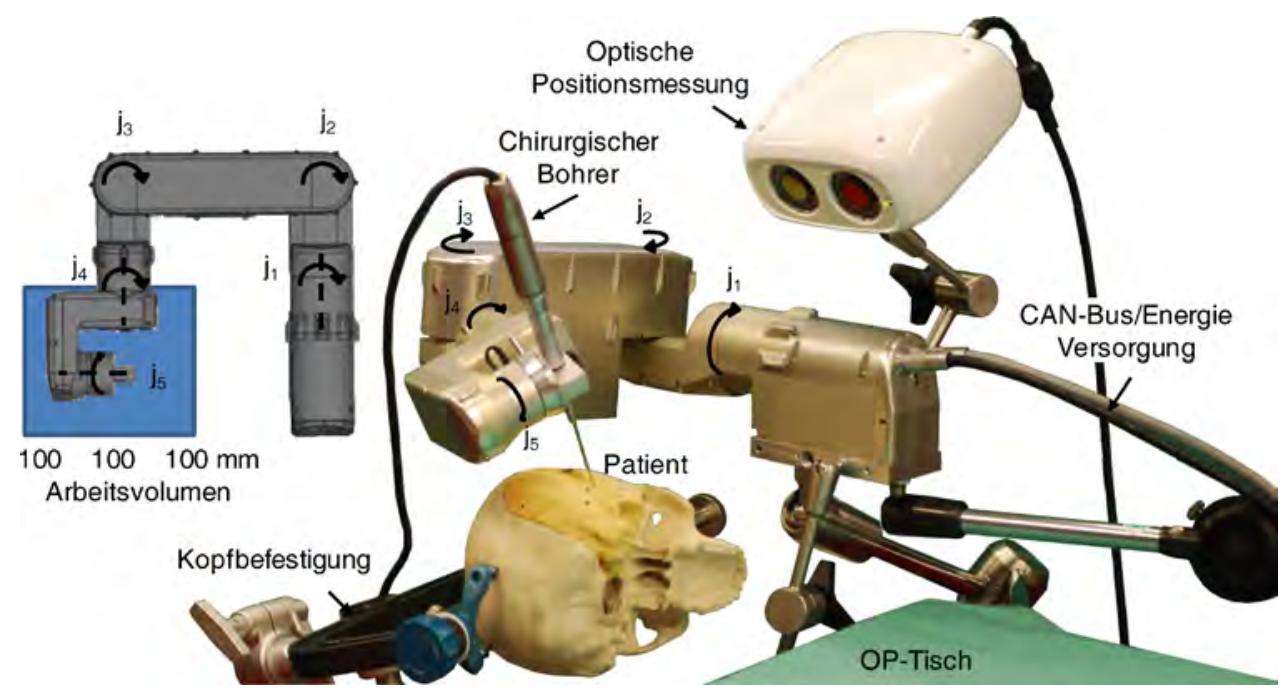

Bild 2 Roboterbasiertes und navigiertes Instrumentenführungssystem mit einer Darstellung des verfügbaren optimalen Arbeitsraumes (links).

werden. Um die erforderliche Aufbauzeit des Roboters im OP und die Anzahl anzuschliessender Kabel zu reduzieren, wurde die Steuerelektronik (Achsregler, CAN-Bus Controller) direkt in den Roboter integriert. Als Antriebe werden Gleichstrommotoren mit einem Drehmoment von 33,3 mNm bei $7100 \mathrm{~s}^{-1}$ (EC 30, Maxon Motor, Schweiz) in den Achsen 1 bis 3 sowie mit $11 \mathrm{mNm}$ bei $8240 \mathrm{~s}^{-1}$ (EC 22, Maxon Motor, Schweiz) in den Achsen 4 bis 5 eingesetzt. Alle Motoren sind mit digitalen Encodern mit einer Auflösung von $0,0005^{-1}$ sowie mit elektrischen Bremsen ausgestattet. Wird die Energiezufuhr zum Roboter unterbrochen, werden die letzten verfügbaren Encoderwerte ausgelesen und nichtflüchtig über einen integrierten Mikrocontroller (DSPIC33, Microchip) gespeichert. Bei einer Inbetriebnahme des Roboters können die Achspositionen initial ausgelesen werden, das Anfahren einer bekannten Nullposition ist nicht erforderlich. Hohe Übersetzungen in den Achsen (560: 1 in den Achsen 1 bis 3 bzw. $400: 1$ in Achsen 4 und 5) konnten durch Spannungswellengetriebe (sogenannte Harmonic Drives, ASS AG, Schweiz) kombiniert mit Zahnriemenantrieben erreicht werden. Dies ermöglicht einen kompakten, leichten sowie spielfreien Aufbau der Achsen. Im Endeffektor des Roboters wurde zusätzlich ein 6D-Kraft-Momentensensor (KMS, Nano 40, ATI Industrial Automation, USA) integriert und ermöglicht so eine haptische Führung und kraftgesteuerte Positionierung chirurgischer Werkzeuge, sowie die Überwachung von Gewebe- und eingriffsspezifischen Kräften und Momenten [7]. In späteren Ausbaustufen des Systems ist die Anwendung des KMS bei der kraftgesteuerten Ausmessung der Knochenschrauben vorgesehen.

\subsection{Optische Positionsmessung}

Ein optisches Positionsmesssytem (Cambar B1, Axios Gmbh) ist an der Basis des Roboters so angebracht, das der Arbeitsraum des Endeffektors am Patienten optisch überwacht und vermessen werden kann. Die Positi- onsmessung der Instrumentenspitze erfolgt dabei mit einer Genauigkeit von $0,01 \mathrm{~mm}$ in einem Arbeitsvolumen von $150 \times 150 \times 150 \mathrm{~mm}^{3}$. Das Messsystem kann dabei sowohl passive als auch aktive optische Referenzen detektieren. Für eine maximale Genauigkeit des Messverfahrens wurden daher eigene LED basierte aktive Referenzen entwickelt, die eine Positionserfassung des Roboters mit einer Messfrequenz von bis zu $30 \mathrm{~Hz}$ ermöglichen (2 Referenzen mit jeweils 4 Messpunkten, Bildverarbeitung auf einem Intel Core2 Quad CPU, 4 GB RAM).

\subsection{Kalibrierung und Genauigkeit}

Die kinematische Kalibrierung des Roboters nach dessen Herstellung wurde mit einer Koordinatenmessmaschine (3D Micro-MS 454, Tesa, Schweiz) durchgeführt. Nach Festlegen eines Basiskoordinatensystems an der Roboterbasis werden die einzelnen Gelenke rechtwinklig zueinander ausgerichtet. Die eingestellten Gelenkpositionen werden mittels spezieller am Gehäuse integrierter Messkugeln digitalisiert. Die Abweichungen zwischen gemessenen und idealen (aus den CAD-Daten) Gelenkpositionen fliessen als Korrekturparameter in das Denavit-Hardenberg Modell der Kinematik ein.

Die effektive absolute Positioniergenauigkeit bestimmt, indem der Roboter an bekannte Positionen im Arbeitsraum verfahren wurde und die Lage des Endeffektors mittels Koordinatenmessmaschine ausgemessen wurde. Die absolute Positioniergenauigkeit beträgt im optimalen Arbeitsraum $\left(100 \times 100 \times 100 \mathrm{~mm}^{3}\right)$ maximal $0,25 \mathrm{~mm}$. Die effektive Wiederholgenauigkeit wurde bestimmt, indem der Roboter wiederholt an die gleiche Position verfahren wurde (ausgehend von der HomePosition, $n=3$ ) und beträgt weniger als $0,06 \mathrm{~mm}$.

Die Flexibilität des Roboters im optimalen Arbeitsraum wurde mittels des hochauflösenden optischen Messsystems (Cambar B1) und bei statischen Belastungen des Endeffektors ermittelt und liegt zwischen 0,01 und 
0,02 $\mathrm{mmN}^{-1}$, abhängig von der jeweiligen Lastrichtung. Die im Vergleich zum Gehäuse geringe Steifigkeit der verwendeten Spannungswellengetriebe trägt dabei zu der ermittelten Gesamtsteifigkeit bei und kann zukünftig bei der Entwicklung weiterer Versionen des Gesamtsystems berücksichtigt werden.

\subsection{Integration im OP}

Der Kopf des Patienten wird während des Eingriffs mittels einer speziellen 3-Punktklemme spielfrei am OPTisch gelagert (FixIt, Medicon GmbH, Deutschland). Der Roboter wird mittels Gelenkarmen (Fisso Baitella AG, Schweiz) am OP-Tisch befestigt und zusätzlich an der Kopfklemme versteift um relative Bewegungen zwischen Roboter und Schädel zu minimieren. Bild 2 zeigt diesen Aufbau des Roboters am OP-Tisch.

\subsection{Navigationsbasierte Robotersteuerung}

Die Lageregelung des Roboters erfolgt über eine Kaskadenregelung. Die oberste Ebene der Regelung wird dabei über das optische Positionsmesssystem realisiert, das die Istposition des Endeffektors unabhängig von den Encodern der einzelnen Achsen erfassen kann. Zusammen mit den im Navigationssystem zur Verfügung stehenden Planungsdaten (Sollpositionen) wird eine übergeordnete Lageregelung realisiert. Zusätzlich wird über diese Form der redundanten Positionserfassung (optisch Messung und mechanisch über Encoder) ein Sicherheitsarchitektur realisiert, die im Falle von Problemen in den Reglern unerwünschte Bewegungen des Roboters sofort erfasst und diesen durch direkten Eingriff in die Spannungsversorgung (Not-Aus) außer Betrieb setzen kann. Zusätzlich arbeiten auf weiteren Ebenen Regler für Positionen und Geschwindigkeiten in den Koordinatensystemen der einzelnen Achsen sowie im kartesischen Basissystem.

Die Bedienung des Gesamtsystems erfolgt über eine intuitive graphische Benutzerschnittstelle (Bild 3) auf dem Navigationssystem. Diese Benutzerschnittstelle strukturiert den Workflow durch einzeln bearbeitbare Schritte bei Inbetriebnahme der Sensorik, der Registrierung der Bilddaten mit dem Patienten und den eigentlichen Bohrund Fräsvorgang (siehe auch Abschnitt 2.3) sowie die notwendigen Instrumentenwechsel. Der Chirurg (der Benutzer) wird über den Touchscreen über die einzelnen Schritte informiert und bestätigt diese. Zusätzlich werden aktuelle Zustandsmeldungen der Teilsysteme (Roboter, optisches Positionsmesssystem, KMS) am Bildschirm angezeigt. Der Benutzungsablauf für die Benutzung des Systems entspricht dabei dem standardisierten Ablauf einer konventionellen Operation und wurde entsprechend den Anforderungen des Navigations- und Robotersystems (Registrierung, Instrumentenwechsel, Instrumentenführung) ergänzt.

Während des eigentlichen Bohr- und Fräsvorgangs wird die aktuelle Instrumentenposition auf dem Bildschirm im Koordinatensystem der medizinischen Bilddaten in einem 3D-Kontext angezeigt. Die durch den

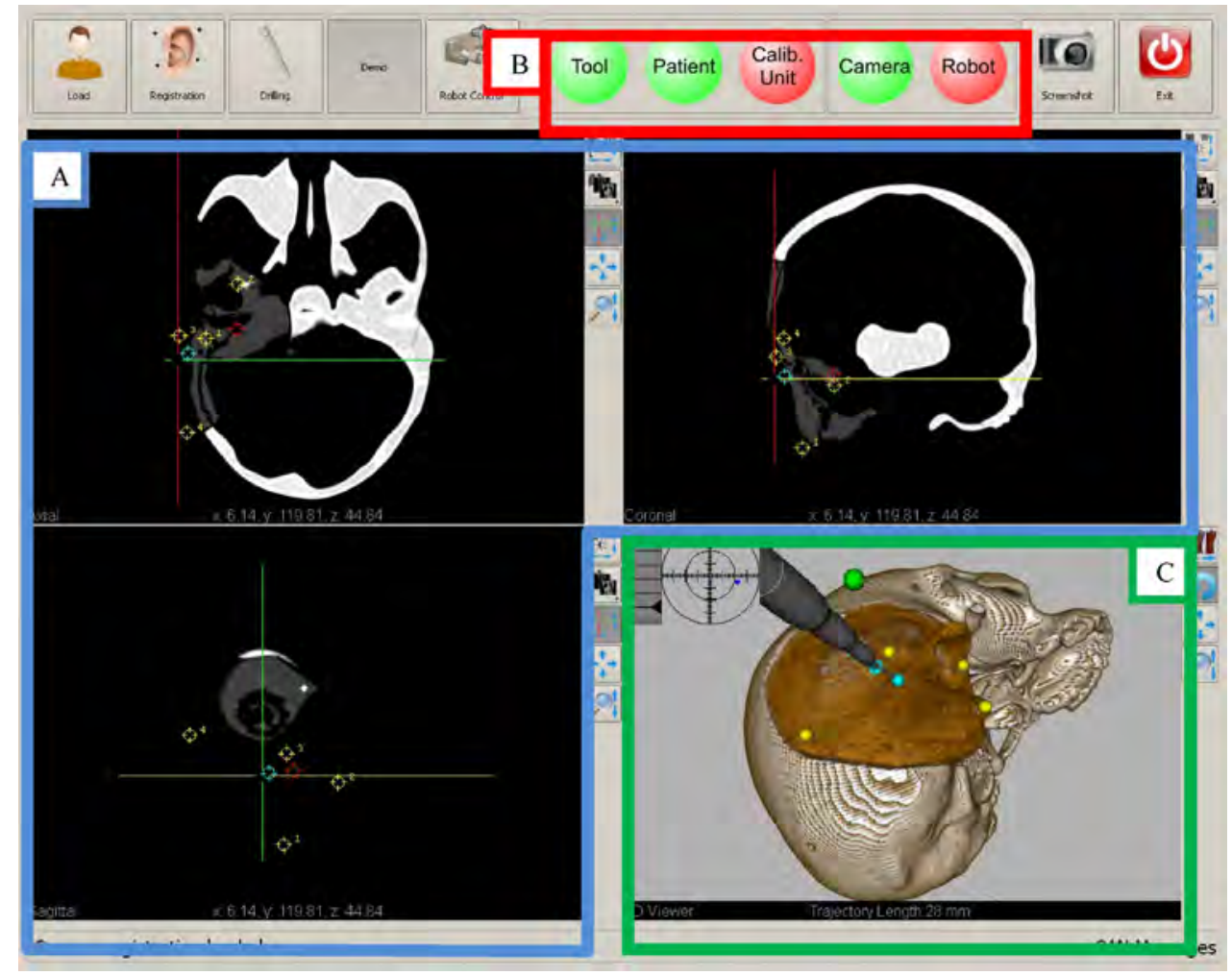

Bild 3 Die graphische Benutzerschnittstelle ist unterteilt in Bereiche für die Darstellung der medizinischen Bilddaten (A), die Anzeige der Statusinformationen der Teilsysteme Roboter und Positionsmesssystem (B) sowie eine 3D Darstellung der Werkzeugposition in Beziehung zu dem Kopf (C). 
Benutzer erfolgten Eingaben werden an die Robotersteuerung über den CAN-Bus geleitet. In der Robotersteuerung werden die Sollvorgaben unter Verwendung der kinematischen Modellierung und einer Trajektorienplanung in Sollpositionen und -geschwindigkeiten für die einzelnen Achsen umgesetzt und an die einzelnen Achsregler (im Roboter) wiederum über CAN-Bus übertragen.

\section{Material und Methoden}

Das Ziel der in diesem Beitrag vorgestellten Arbeiten besteht in der Realisierung patientenindividueller Implantatlager aus komplexen Fräßgeometrien im Knochengewebe des Schädels. Diese komplexen Fräsgeometrien sind zum Einen wichtig für einen passgenauen Sitz von Implantaten im Knochen, zum Anderen müssen die Lage und Position dieser Implantatlager individuell an die antomischen Gegebenheiten des Patienten angepasst werden. Als Grundlage dieses Experiments wird ein Plastikmodell der Felsenbeinregion, wie es für die chirurgische Ausbildung eingesetzt wird (www.temporalbone.com, Großbritannien) verwendet. Dieses Phantom besteht aus den knöchernen Bestandteilen der Felsenbeinregion, den knöchernen anatomischen Strukturen im Mittelohr (Ossicula auditus) sowie einem (nur im CTBild sichtbaren) Verlauf des Gesichtsnervs. Anhand dieses Felsenbeinmodells und eines dreidimensionalen Bilddatensatzes wurde das Implantatlager zunächst geplant und mittels Roboterunterstützung gefräst. Für diesen Versuch wurde auf die oberste Ebene der Lageregelung durch die optische Messkamera aus Genauigkeitsgründen verzichtet.

\subsection{Workflow für das Fräsen einer vordefinierten Implantatgeometrie}

Der angewandte und klinisch übertragbare Workflow ist in Bild 4 im Überblick dargestellt und besteht aus den folgenden Schritten:

Implantation von Knochenschrauben für die Referenzierung. Für eine präzise Referenzierung des 3DBilddatensatzes der Patientenanatomie mit dem Patienten werden Knochenschrauben aus Titan $(1.5 \times 5 \mathrm{~mm}$, Medartis AG Schweiz) mit einem sphärischen $4 \mathrm{~mm}$ Schraubenkopf aus Titan als künstliche Landmarken verwendet. Diese knochenverankerten Schrauben sind klinisch als Goldstandard bei der Referenzierung akzeptiert und stellen derzeit das Verfahren mit der höchsten Genauigkeit bei der Referenzierung dar [8].
Sie sind außerdem im Vergleich zum umgebenden Knochen aufgrund ihrer höheren Dichte (Titan gegenüber Knochengewebe) gut abgrenz- und damit segmentierbar. Diese Schrauben werden vor der Bildgebung an vier verschiedenen Stellen um das Operationsgebiet am Ohr implantiert. Die Schraubenpositionen und -orientierungen der Knochenschrauben werden in den aufgezeichneten Bilddaten danach automatisch bestimmt [9]. Die Positionen der Schrauben am Kopf werden durch das Robotersystem digitalisiert und über ein Pair-Point-Matching verfahren mit den Positionen im Bilddatensatz referenziert.

Bildgebung. Grundsätzlich tragen hochauflösende und qualitativ aussagekräftige Bildgebungsverfahren zur Erhöhung der Genauigkeit in der computerassistierten Chirurgie bei [10]. So konnten wir bereits zeigen, dass der Einsatz volumentomographischer Verfahren für die Durchführung von bildgeführten Eingriffen am Ohr hinsichtlich Strahlenbelastung, räumlicher Auflösung und radiologisch nutzbarer Bildqualität einfachen computertomographischer Verfahren überlegen ist [11] und außerdem eine Verbesserung der Lokalisation der Knochenschrauben ermöglicht [9]. In der vorliegenden Studie werden daher als Grundlage für die Eingriffsplanung und -auswertung DVT Daten verwendet. Der resultierende Datensatz mit einer Auflösung von $0,15 \times 0,15 \times$ $0,15 \mathrm{~mm}^{3}$ steht für die Eingriffsplanung zur Verfügung.

Softwarebasierte Operationsplanung. Für die Eingriffsplanung wurde ein eigenständiges Softwaremoduel OtoPlan entwickelt, dass auf der Basis präzise identifizierter anatomischer Strukturen eine Planung der späteren Implantatlage und -orientierung ermöglicht (Bild 5). Dazu wird der Bilddatensatz in die Software eingelesen und in einem ersten Schritt die Region des Felsenbeins über Schwellwerte segmentiert, danach der Gesichtsnerv sowie die Paukenseite manuell eingezeichnet. Das manuelle Vorgehen stellt dabei sicher, dass die Nerven möglichst präzise und mit einem Sicherheitsabstand identifiziert werden, und wird als Goldstandard angenommen [12]. Zusätzlich werden die Gehörknöchelchen über einen Region-Growing Ansatz identifiziert und automatisch segmentiert. Die Genauigkeit der automatischen Segmentierung reicht hier im Gegensatz zu den Nerven aus, da diese weiter von der Trajektorie entfernt sind. Zusätzlich werden die Knochenschrauben segmentiert und deren Lage im Koordinatensystem der Bilddaten über eine Minimierung des Abstandes zwischen der

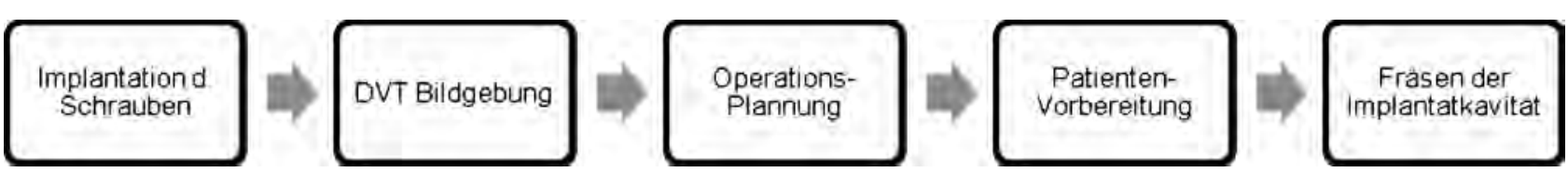

Bild 4 Workflow zur robotergestützen Realisierung eines patientenindividuellen Implantatlagers. 


\section{Anwendungen}

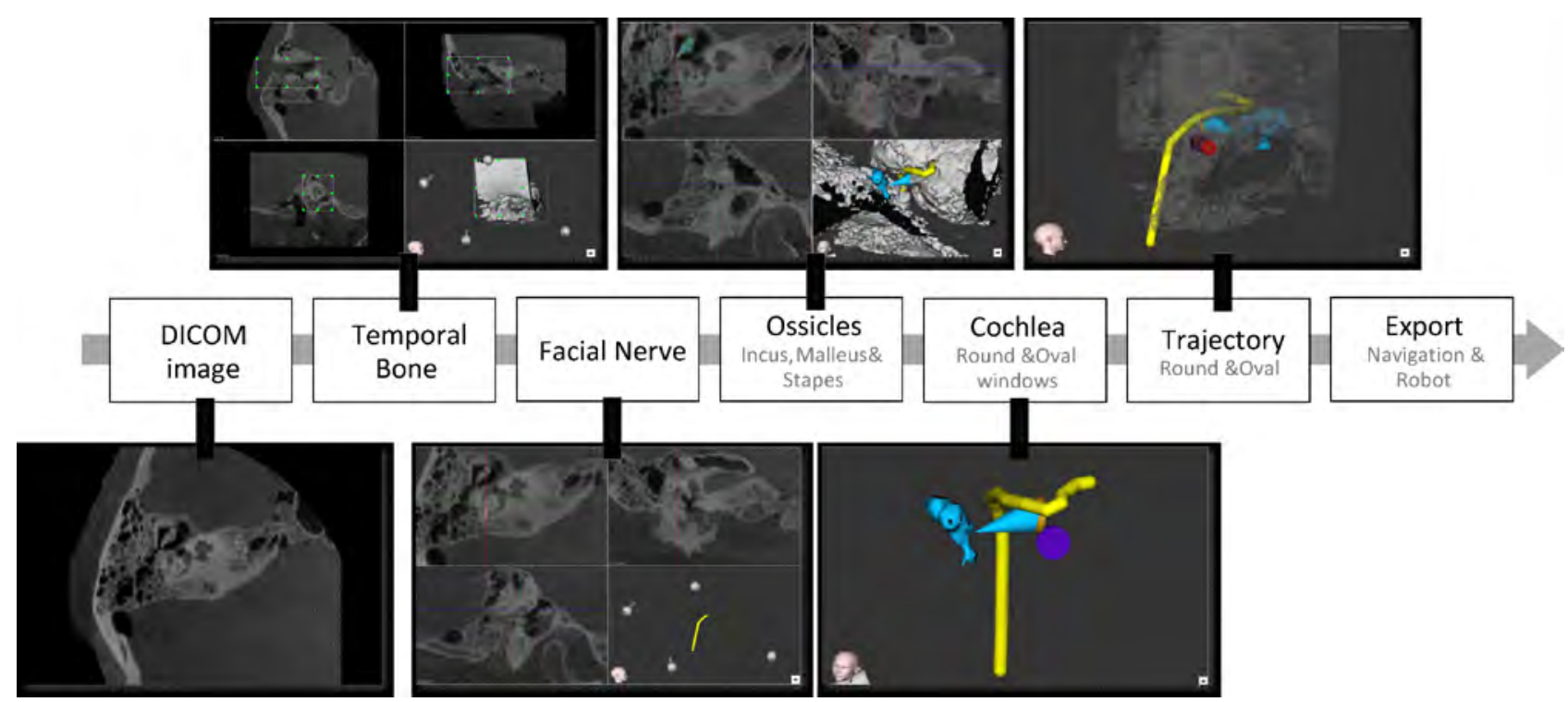

Bild 5 Schematischer Ablauf der computerbasierten Planung im softwarebasierten Planungsmodul Otoplan.

Schwellwertsegmentierung und dem eigentlichen CADModell der Schrauben ermittelt [9].

Planung der Implantkavität. Nach der Segmentierung der relevanten anatomischen Strukturen wird die Lage des Implantats ausgehend vom runden Fenster der Cochlea (Zielpunkt) und zwischen einem schmalen Fenster zwischen Gesichtsnerv und Paukensaite (Facial Recess) hindurch festgelegt. Damit ergeben sich Eintritts- und Zielpunkte für eine Trajektorie entlang der späteren Implantatlage (Bild 6a). Ausgehend von dieser Trajektorie und einer definierten Implantatgeometrie für ein neuartiges Mittelohrimplantat zur direkten Stimulation der Cochlea (Direct Acoustic Cochlea Stimulation [13]) wurde die zu fräsende Kavität geplant. Die Implantatgeometrie besteht aus drei konzentrisch zueinander angeordneten Zylindern mit unterschiedlichen Längen und Durchmessern. Zusätzlich befindet sich proximalen noch eine zusätzliche Einbuchtung zur Aufnahme eines Befestigungsankers (siehe Tabelle 2).

Daraus werden für vordefinierte chirurgische Fräser spiralförmige Helixtrajektorien in unterschiedlichen Tiefen abgeleitet. In Bild 6b ist die geplante Frästrajektorie dargestellt. Die Stützpunkte der Helixtrajektorien $(\Delta=0,1 \mathrm{~mm})$ werden durch die Pfadplanung (mittlere Ebene der Kaskadenregelung) der Roboterregelung berechnet und zusammen mit Geschwindigkeitssollwerten an die Achsregler übergeben. Um eine schnelle und effiziente Bearbeitung zu gewährleisten wurde mit zwei verschiedenen Werkzeugen gearbeitet (siehe Tabelle 2). In einem ersten Schritt wird mit einem $4 \mathrm{~mm}$ Rosenkopffräser die grobe Struktur ausgefräst (siehe Bild 6,

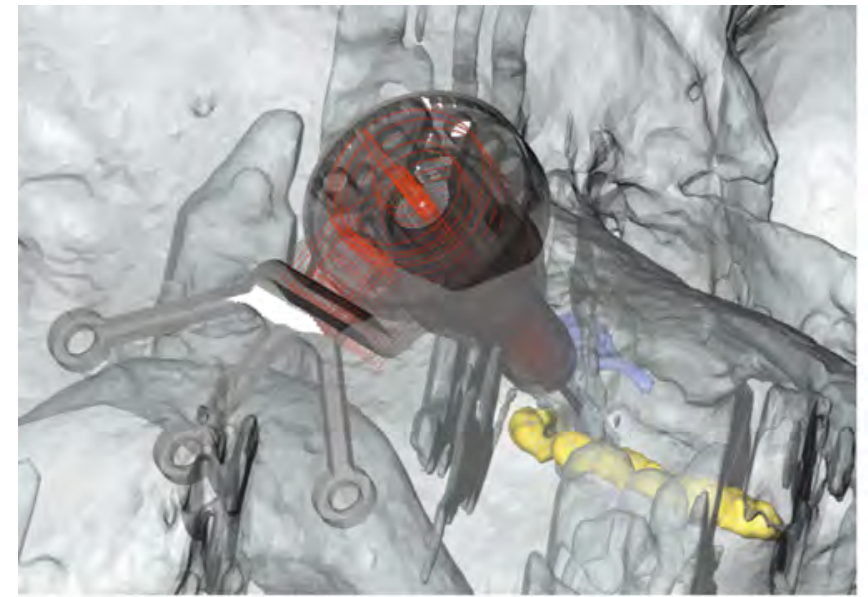

a)

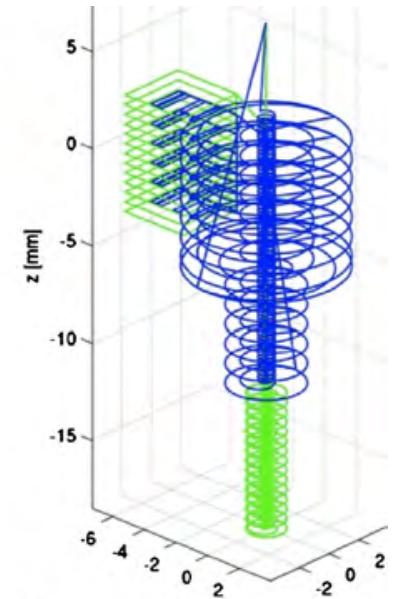

b)

Bild 6 (a) Geplante Implantatlage des DACS Implantats im Felsenbein, relativ zum Gesichtsnerv (gelb) und den Gehörknöchelchen und dem Zielpunkt des runden Fensters in der Cochlea. Sichtbar ist ebenfalls die geplante Trajektorie (rot); (b) Die Kavität wird als zusammenhängende Trajektorie mit verschiedenen Fräsdurchmessern gefräst. 
Tabelle 2 Parameter der Implantatgeometrie und Werkzeugeigenschaften.

\begin{tabular}{lcccc}
\hline Struktur & Länge in mm & Durchmesser in mm & Grobfräsung* & Feinfräsung** \\
\hline Zylinder & 6 & 11,9 & Rosenkopffräser $4 \mathrm{~mm}$ & Rosenkopffräser 2 mm \\
Zylinder & 6 & 7,8 & Rosenkopffräser $4 \mathrm{~mm}$ & $\begin{array}{l}\text { Rosenkopffräser } 2 \mathrm{~mm} \\
\text { Zylinder }\end{array}$ \\
Befestigung & 7,5 & 4,2 & & Rosenkopffräser $2 \mathrm{~mm}$ \\
& \multicolumn{2}{c}{$6,5 \times 3,7 \times 4 \mathrm{~mm}^{3}$} & & Rosenkopffräser $2 \mathrm{~mm}$ \\
\hline
\end{tabular}

* Vorschub: Axial: 0,9 mm/s, Radial: 0,5 mm/s; Abtrag: Axial: $1 \mathrm{~mm}$, Radial: $2 \mathrm{~mm}$

** Vorschub: Axial: 0,45 mm/s, Radial: 0,35 mm/s; Abtrag: Axial: 0,3 mm, Radial: $1 \mathrm{~mm}$

blaue Trajektorie). Aufgrund des grösseren Werkzeugdurchmessers können grössere Vorschübe bei größeren Angriffsflächen erreicht werden, jedoch ergeben sich grössere Radien in den Ecken der Kavitäten. Anschliessend wird mit einem $2 \mathrm{~mm}$ Rosenkopffräser die finale Geometrie der Kavität gefräst (siehe Bild 6, grüne Trajektorie). Dabei sind die Vorschübe und die Eingriffsfläche deutlich geringer, jedoch werden kleine Radien in den Ecken erreicht, um einen passgenauen Sitz für das Implantat zu gewährleisten.

\subsection{Ablauf einer robotergestützten Intervention}

Für eine maximal starre Befestigung wird der Schädel in einer chirurgischen Kopfbefestigung (FixIT, Medicon Instrumente, Deutschland) am OP-Tisch fixiert. Der Roboter wird ebenfalls am OP-Tisch fixiert und so ausgerichtet, dass die Roboterbasis horizontal seitlich versetzt und vertikal auf gleicher Höhe (relativ zur Mitte des Kopfes) ausgerichtet ist. Das verwendete Felsenbeinphan- tom musste dabei aufgrund seiner geringen Grösse (im Vergleich zu einem Schädel) zusätzlich fixiert werden. Der Roboter wurde in die Arbeitsposition verfahren, wobei sich die Instrumentenspitze jetzt oberhalb des Eingriffsgebiets am Felsenbein befindet. Folgender Workflow wird interaktiv mit dem Roboter und per Steuerung über die Benutzerschnittstelle des Navigationssystems ausgeführt wie in Bild 7 dargestellt wird:

1. Referenzierung der Patientenposition: Am Endeffektor des Roboters wird eine Messspitze eingesetzt und unter visueller Kontrolle auf den Schraubenköpfen positioniert. Die Positionen der Schrauben im Koordinatensystem des Roboters werden erfasst.

2. Ausführen einer Zentrierbohrung: Nach Einsetzen eines kurzen und damit sehr steifen Bohrers ( $l=2 \mathrm{~mm}, d=2 \mathrm{~mm}$ ) führt der Roboter eine kurze Zentrierbohrung (Tiefe: $2 \mathrm{~mm}$ ) entlang der geplanten Implantatachse aus. Damit wird sichergestellt, dass sich beim späteren Bohrvorgang der relativ lange Boh-

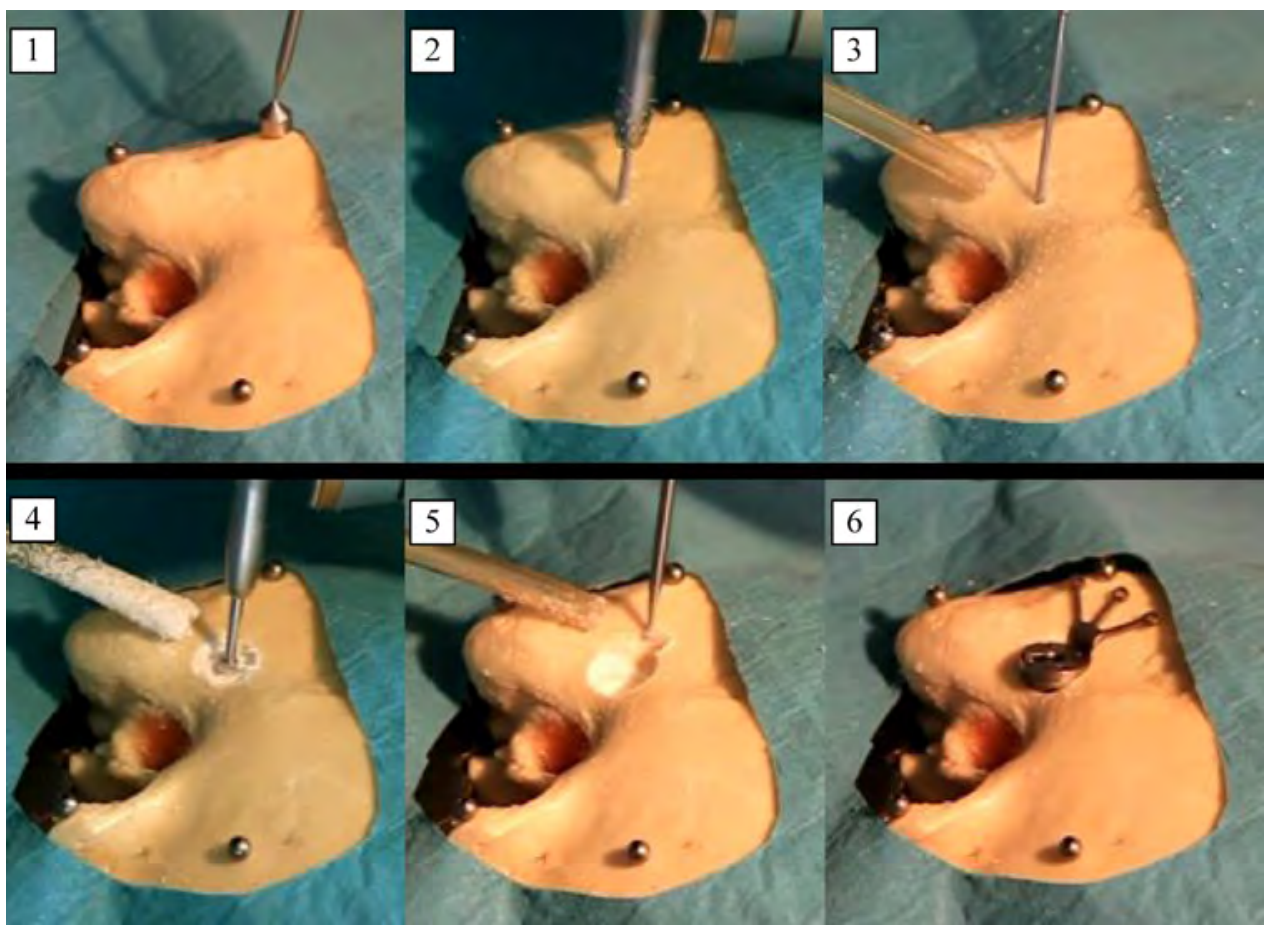

Bild 7 Schritte des Bohr- und Fräsvorgangs: (1) Ausmessen der Positionen der Landmarken, (2) Anfertigen einer Zentrierbohrung, (3) Realisierung des Tunnelzugangs in das Innenohr, (4) Fräsen der Implantatkavität mit $4 \mathrm{~mm}$ Fräser, (5) Feinausfräsung mit 2 mm Fräser, (6) Montiertes Implantat. 
rer für die Tunnelbohrung nicht verwindet, wenn die Knochenoberfläche nicht genau senkrecht getroffen wird.

3. Bohren des Tunnelzugangs: Nach Einsetzen des eigentlichen Bohrwerkzeuges für die Tunnelbohrung ( $l=50 \mathrm{~mm}, d=2 \mathrm{~mm}$ ) richtet sich der Roboter an der geplanten Implantatachse aus und bewegt sich zunächst zu einem Punkt oberhalb des geplanten Eintrittspunktes am Felsenbein. Visuell wird jetzt noch einmal durch den Benutzer die Korrektheit der Ausrichtung überprüft und der Bohrvorgang gestartet. Der Bohrer bewegt sich mit einer Vorschubgeschwindigkeit von $0,1 \mathrm{mms}^{-1}$ und wird durch eine Spülung gekühlt. Nach Abschluss des Bohrvorgangs fährt der Roboter zur Werkzeugwechselposition.

4. Fräsen der Implantatgeometrie: Nach Einsetzen des Fräswerkzeuges mit $5 \mathrm{~mm}$ Werkzeugdurchmesser werden zunächst die größeren (lateralen) Zylinder gefräst.

5. Feinausfräsung: Nach einem Wechsel zum kleineren Fräswerkzeug ( $2 \mathrm{~mm}$ Werkzeugdurchmesser) wird der innere kleinere Zylinder realisiert. Abschließend wird die zusätzliche Kavität für das Befestigungssystem an der Knochenoberfläche hergestellt.

6. Montage des Implantats: Das Implantat wird in die Kavität eingesetzt und mittels des vorhandenen Fixierungssystems im Knochen verschraubt.

7. Postoperative Auswertung: Für die Validierung der korrekten Lage des Bohrloches und der Implantatkavität wird im Anschluss an operativen Eingriff das Phantom erneut im DVT vermessen. Über die vorhandenen Schraubenpositionen werden die prä- und postoperativen Datensätze miteinander registriert und die Abweichung der geplanten zur tatsächlich vorhandenen Lage und Größe der realisierten Kavität wird bestimmt.

\section{Ergebnisse}

\subsection{Kliniktaugliches System} für die computerassistierte Mikrochirurgie

Im Gegensatz zu speziell adaptierten Industrierobotern $[6 ; 7]$ wurde die hier beschriebene Roboterplattform epxlizit für die Anforderungen der HNO-Chirurgie entwickelt. Auf die spätere Integrierbarkeit in die notwendigen klinischen Abläufe (Aufbau am OP-Tisch, steriles Verpacken, Zugang für Operateur und zusätzliche Bildgebung) wurde dabei eingegangen. Das geringere Gewicht ermöglicht die Montage durch das OP-Personal und ohne Unterstützung einer zusätzlichen Hebevorrichtung. Die direkt im Roboter integrierte Leistungs- und Steuerungselektronik erlaubt die Ansteuerung über zwei Kabel mit kleinen Querschnitten. Die Realisierung einer seriellen Kinematik mit einem länglichen Formfaktor erlaubt es, im OP vorhandene sterile Abdeckungen in Schlauchform zu verwenden, die einfach über den Roboter gezogen werden können. Das Bohr/Fräsinstrument (als einzige sterile Komponente) wird von außen auf den Instrumentenanschluss am Roboter montiert. Die vorgesehene Montage

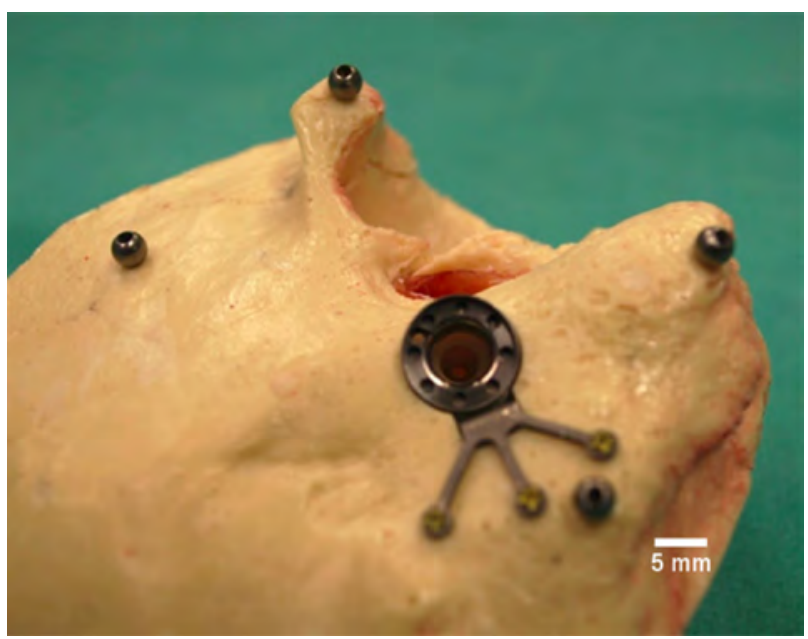

Bild 8 Implantatgehäuse eingesetzt in die gefräste Öffnung im Felsenbein.

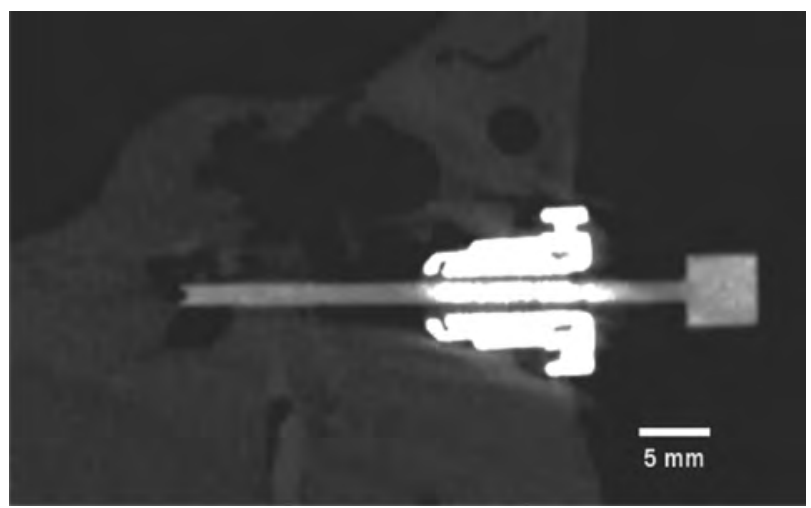

Bild 9 Axialer Schnitt durch Felsenbein und Implantat. Die Spielpassung resultiert in einem sichtbaren Winkel zwischen der Implantatachse (grauer Stift) und der Achse des Implantatgehäuses (hell).

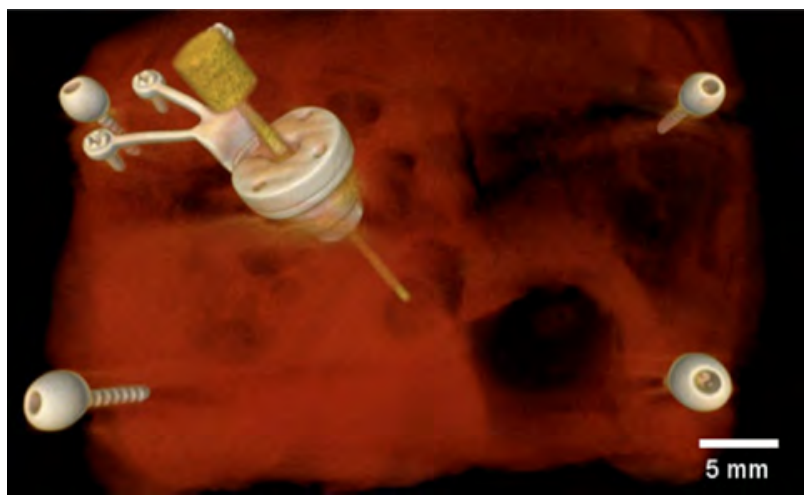

Bild 10 Volumenrendering des eingesetzten Implantats und der Knochenschrauben (hell) im Felsenbein (rot).

am OP-Tisch und die geringe Größe des Roboters auf der Instrumentenseite ermöglichen einen ausreichend großen Zugang des Operateurs in den Situs. Die Verwendung eines OP-Mikroskops ist ebenfalls sichergestellt. Die graphischen und haptischen Eingabeschnittstellen stellen ebenfalls wesentliche Komponenten eines optimalen klinischen Workflows dar, da sie den Chirurg gezielt durch die notwendigen Schritte bei der Plannung und Ausführung durchführen. 
4.2 Fräsen einer Implantatkavität in einem Phantom Die Machbarkeit des vorgeschlagenen Präzisionsworkflows bestehend aus DVT-Bildgebung, computergestützter Planung und roboterassistierter Umsetzung konnte am Beispiel eines mehrteilig gefrästen Implantatlagers in einem anatomischen Modell erfolgreich nachgewiesen werden. Das Fräsen der Kavität unter Verwendung verschiedener Werkzeuge wurde erfolgreich durchgeführt. Der gesamte Registrierungs-, Bohr- und Fräsvorgang dauerte $48 \mathrm{~min}$. Die Verwendung verschiedener notwendiger Bohr- und Fräswerkzeuge mit den notwendigen Vorschubparamatern erfolgte entsprechend der Planung des Implantatlagers. Anschliessend konnte das Implantat erfolgreich implantiert und über die vorhandene Befestigungsmöglichkeit fixiert werden. Da das Implantatlager als Spielpassung ausgeführt wurde, lässt sich das Implantat leicht einsetzen und montieren.

\section{Diskussion}

In dieser Arbeit wurde der Einsatz eines roboterbasierten Instrumentenführungssystems für das Fräsen bei chirurgischen Eingriffen zur Implantation von Hörgeräten vorgestellt. Das System wurde anhand eines geplanten und mittels Roboters umgesetzten Fräsvorganges für eine Implantattasche validiert. Das Gesamtsystem (bestehend aus Roboter, Steuerung und notwendigen Softwaremodulen) wurde im Sinne der optimalen klinischen Integrierbarkeit entworfen und unter klinischen Bedingungen validiert. Für die Gebrauchstauglichkeit des Roboters stehen sein Gewicht, die einfache Montage im $\mathrm{OP}$ und die einfache sterile Verpackung. Die intuitive Steuerung des Roboters durch eine interaktive Benutzersteuerung ermöglicht eine einfache Bedienung direkt durch den Operateur.

Das Hauptziel dieser Arbeit war der Machbarkeitsnachweis des Fräsens einer beliebigen und im Kontext medizinischer 3D Bilddaten geplanter Implantatkavität. Dies kann künftig die Implantation von spezifischen Hörgeräten mit komplexen Gehäusegeometrien vereinfachen und präzisieren. Interessant erscheint außerdem die Möglichkeit einer Vereinfachung der Implantatgeometrien, wenn diese mit einem Roboter umgesetzt werden können. Gegenwärtige Implantatdesigns wurden bisher nur unter dem Gesichtspunkt einer manuellen Implantation entworfen, sind aber vielfach noch optimier- und reduzierbar. Diese Möglichkeit ist auch vor dem Hintergrund einer möglichst präzisen Implantatpositionierung interessant. In der vorliegenden Arbeit wurde die zu realisierende Kavität absichtlich nicht passgenau realisiert um eine einfache Montage des Implantatlagers im Felsenbein $\mathrm{zu}$ ermöglichen. Auf die Optimierung der erforderlichen Fräszeit (48 min) wurde in dieser Studie (noch) kein Wert gelegt und die Fräßgeschwindigkeit so gewählt, dass der Fräsvorgang ohne größere Krafteinleitungen und damit einhergehende Verwindungen von Roboter und Kopfbefestigung einhergeht. Eine Optimierung der Arbeitsgeschwindigkeit stellt aber eine klare Herausforde- rung zukünftiger Arbeiten dar. Dies soll durch die Suche nach einem zweckmässigen Frässystems bei gleichzeitiger Sicherstellung der geeigneten Fräskraft erfolgen. Durch die relativ geringe Eigensteifigkeit des Roboters - bedingt unter Anderem durch die serielle Kinematik - können bereits kleine Fräskräfte bedeutende Verformungen hervorrufen. In der aktuellen Implementierung findet derzeit kein Kompensation der am Robotersystem anliegenden Kräfte und Momenten statt.

\section{Danksagung}

Die Autoren danken Simon Roder und Jonas Salzmann für ihre technische Unterstützung des Roboterprojekts. Ins besondere für das Programmieren der Steuerung und technische Beiträge. Wir wollen Einar Nielsen und Dominik Widmer von der Interstaatliche Hochschule Buchs, Schweiz danken für die Hilfe die sie während der Designphase des Roboters geleistet haben. Zum Schluss sagen wir Urs Rohrer Danke für die werkstattliche Hilfe.

\section{Literatur}

[1] J. Schipper et al., Navigation as a quality management tool in cochlear implant surgery. In: The Journal of Laryngology and Otology, vol. 118, no. 10, pp. 764-770, Oct. 2004.

[2] K. Cleary and T.M. Peters, Image-guided interventions: technology review and clinical applications. In: Annual review of biomedical engineering, vol. 12, pp. 119-42, Aug. 2010.

[3] B. Bell et al., An accuracy approach to robotic microsurgery in the ear. In: 9. Jahrestagung der Deutschen Gesellschaft für Computerund Roboterassistierte Chirurgie, pp. 77-82, 2010.

[4] O. Majdani et al., A robot-guided minimally invasive approach for cochlear implant surgery: preliminary results of a temporal bone study. In: International journal of computer assisted radiology and surgery, vol. 4, no. 5, pp. 475-486, Sep. 2009.

[5] S. Baron et al., Percutaneous inner-ear access via an imageguided industrial robot system. In: Proceedings of the Institution of Mechanical Engineers, Part H: Journal of Engineering in Medicine, vol. 224, no. 5, pp. 633-649, 2010.

[6] T. Klenzner et al., New strategies for high precision surgery of the temporal bone using a robotic approach for cochlear implantation. In: European Archives of Oto-Rhino-Laryngology, vol. 266, no. 7, pp. 955-960, 2009.

[7] P. N. Brett, Moving on from surgical robotics to robotic microtools in surgery, In: 2007 14th International Conference on Mechatronics and Machine Vision in Practice. Ieee, pp. 1-5, Dec-2007.

[8] C. R. Maurer, J. M. Fitzpatrick, M.Y. Wang, R. L. Galloway, R. J. Maciunas, and G.S. Allen, Registration of head volume images using implantable fiducial markers, In: IEEE transactions on medical imaging, vol. 16, no. 4, pp. 447-62, Aug. 1997.

[9] G. Zheng et al., Automated detection of fiducial screws from CT/DVT volume data for image-guided ENT surgery.," Conference proceedings: ... Annual International Conference of the IEEE Engineering in Medicine and Biology Society. IEEE Engineering in Medicine and Biology Society. Conference, vol. 1, pp. 2325-8, Jan. 2010.

[10] S.H. Bartling et al., Increase of accuracy in intraoperative navigation through high-resolution flat-panel volume computed tomography: experimental comparison with multislice computed tomography-based navigation. In: Otology \& Neurotology, vol. 28, no. 1, p. 129, Jan. 2007.

[11] B. Bell et al., Improving System Accuracy in Computer Aided Robotic ORL Surgery. In Hamlyn Symosium on Medical Robotics, pp. 11-12, 2010. 
[12] J. H. J. H. Noble, O. Majdani, R. F. Labadie, B. Dawant, and J. M. M. Fitzpatrick, Automatic determination of optimal linear drilling trajectories for cochlear access accounting for drill-positioning error, The International Journal of Medical Robotics and Computer Assisted Surgery, vol. 6, no. 3, pp. 281-290, Sep. 2010.

[13] R. Häusler, C. Stieger, H. Bernhard, and M. Kompis, A novel implantable hearing system with direct acoustic cochlear stimulation, Audiology \& neuro-otology, vol. 13, no. 4, pp. 247-56, Jan. 2008.

Manuskripteingang: 3. März 2011

Brett J. Bell leitet die Robotik-Gruppe der Institut für Chirurgische Technologie an der Universität Bern seit 2010. Er hat in 2003 an der Utah State University einen Bachelor of Science in Maschinenbau und später in 2009 einen Ph.D. in Biomedical Engineering an der Purdue University abgeschlossen. Seine Forschungsinteressen liegen bei Chirurgische Instrumenten und Robotik.

Adresse: Institut für chirurgische Technologien und Biomechanik, Universität Bern, Schweiz

Nicolas Gerber verfolgt seinen Ph.D. in Biomedical Engineering an der Universität Bern. Zuvor absolvierte er einen Bachelor of Science in Microengineering mit Fachrichtung Mechatronik an der Universität für Angewandten Wissenschaften der Westlichen Schweiz im Jahr 2007 und einen Master of Science in Biomedical Engineering mit Fachrichtung Mikrosensor- und Antriebstechnologie an der Universität Bern im Jahr 2009.

Adresse: Institut für chirurgische Technologien und Biomechanik, Universität Bern, Schweiz

Kate A. Gavaghan verfolgt ihre Ph.D. in Biomedical Engineering an der Universität Bern. Zuvor hat sie eine Studium in Informatik von der University of Melbourne, in 2003 und Ingenieur Wissenschaft von der Swinburne University of Technology, Melbourne, Australien im
2009 abgeschlossen. Ihre Forschungsinteressen umfassen visuelles und haptisches Feedback für Virtual und Augmented Reality-Schnittstellen für Computer-gestützte Chirurgie.

Adresse: Institut für chirurgische Technologien und Biomechanik, Universität Bern, Schweiz

Christoph Stieger hat an der ETH Zürich Elektrotechnik und Biomedizinische Physik studiert und abgeschlossen. Im Jahre 2006 wurde ihm der Doktortitel am Institut für Mikrotechnik der Universität Neuenburg, Schweiz, verliehen und er leitete die Audiologische Station ad interim. Im Jahre 2008 wurde er zum Assistenzprofessor am ARTORG Center der Universität ernannt und leitet die Forschungsgruppe Artificial Hearing Research.

Adresse: ARTORG Zentrum für Biomedizinische Forschung, Universität Bern, Schweiz und Universitätsklinik für Hals-, Nasen- und Ohrenkrankheiten, Inselspital Bern, Schweiz

Prof. Dr. Med. Marco Caversaccio hat sein Staatsexamen 1990 in Genf und Promotion 1993 mit der Arbeit: Les hématomes intracérébraux spontanés supratentoriels abgeschlossen. Er ist seit 2009 Professor der HNO Chirurgie an der Universitäts Spital in Bern, Schweiz. Professor Caversaccio interesiert sich für Computerassistierte-Chirurgie im Bereich $\mathrm{HNO}$.

Adresse: Universitätsklinik für Hals-, Nasen- und Ohrenkrankheiten, Inselspital Bern, Schweiz

Stefan Weber ist Ko-Direktor des ARTORG Centers für computerassistierte Chirurgie an der Universität Bern. Er studierte Elektrotechnik und Robotik an der TU Ilmenau und an der University of Southern California, Los Angeles USA. Er promovierte am Berliner Zentrum für mechatronische Medizintechnik an der Humboldt-Universität zu Berlin und war als Gruppenleiter am Lehrstuhl für Mikro- und Medizingerätetechnik an der TU München tätig.

Adresse: Institut für chirurgische Technologien und Biomechanik, Universität Bern, Schweiz und ARTORG Zentrum für Biomedizinische Forschung, Universität Bern, Schweiz

\section{Vorschau auf Heft 11/2011}

Unsere nächste Ausgabe ist ein Schwerpunktheft zum Thema „Automatisierungstechnische Verfahren und Systeme für die Medizin, Teil II“ (Gastherausgeber: R. Riener, O. Simanski) und enthält unter anderem folgende Beiträge:

- Boll, M.-T. et al.: Entwicklung eines Bewertungssystems für VR-Simulatoren in der minimal invasiven Chirurgie

- Pylaiuk, C. et al.: Ingenieurtechnische Besonderheiten bei der automatischen Handhabung von biologischen Organismen

- Malberg, H. et al.: Kardiovaskuläre Variabilitätsanalysen zur Risikostratifizierung nach Herzoperationen

- Simanski, O. et al.: Modellbasierte Regelung pneumatisch betriebener Herzunterstützungssystem

Weitere Informationen über geplante Hefte, ausführliche Informationen über die in den letzten Heften der at erschienenen Beiträge sowie Hinweise für Autoren finden Sie im Internet unter http://www.at-automatisierungstechnik.de. 